\title{
On some generalized $q$-Eulerian polynomials
}

\author{
Zhicong Lin \\ Department of Mathematics and Statistics, Lanzhou University \\ Lanzhou 730000, P.R. China \\ Université Lyon 1; Institut Camille Jordan; UMR 5208 du CNRS \\ 43, boulevard du 11 novembre 1918, F-69622 Villeurbanne Cedex, France \\ lin@math.univ-lyon1.fr \\ Submitted: Nov 28, 2012; Accepted: Mar 2, 2013; Published: Mar 8, 2013 \\ Mathematics Subject Classifications: 05A05, 05A19
}

\begin{abstract}
The $(q, r)$-Eulerian polynomials are the (maj-exc, fix, exc) enumerative polynomials of permutations. Using Shareshian and Wachs' exponential generating function of these Eulerian polynomials, Chung and Graham proved two symmetrical $q$-Eulerian identities and asked for bijective proofs. We provide such proofs using Foata and Han's three-variable statistic (inv-lec, pix, lec). We also prove a new recurrence formula for the $(q, r)$-Eulerian polynomials and study a $q$-analogue of Chung and Graham's restricted descent polynomials. In particular, we obtain a generalized symmetrical identity for these restricted $q$-Eulerian polynomials with a combinatorial proof.
\end{abstract}

Keywords: Eulerian numbers; symmetrical Eulerian identities; hook factorization; descents; admissible inversions; permutation statistics

\section{Introduction}

The Eulerian polynomials $A_{n}(t):=\sum_{k=0}^{n} A_{n, k} t^{k}$ are defined by the exponential generating function

$$
\sum_{n \geqslant 0} A_{n}(t) \frac{z^{n}}{n !}=\frac{(1-t) e^{z}}{e^{z t}-t e^{z}}
$$

The coefficients $A_{n, k}$ are called Eulerian numbers. The Eulerian numbers arise in a variety of contexts in mathematics. Let $\mathfrak{S}_{n}$ denote the set of permutations of $[n]:=\{1,2, \ldots, n\}$. For each $\pi \in \mathfrak{S}_{n}$, a value $i, 1 \leqslant i \leqslant n-1$, is an excedance (resp. descent) of $\pi$ if $\pi(i)>i($ resp. $\pi(i)>\pi(i+1))$. Denote by $\operatorname{exc}(\pi)$ and $\operatorname{des}(\pi)$ the number of excedances 
and descents of $\pi$, respectively. It is well-known that the Eulerian number $A_{n, k}$ counts permutations in $\mathfrak{S}_{n}$ with $k$ descents (or $k$ excedances), that is

$$
A_{n}(t)=\sum_{\pi \in \mathfrak{S}_{n}} t^{\operatorname{des} \pi}=\sum_{\pi \in \mathfrak{S}_{n}} t^{\operatorname{exc} \pi}
$$

The reader is referred to $[9,22]$ for some leisurely historical introductions of Eulerian polynomials and Eulerian numbers.

Several $q$-analogs of Eulerian polynomials with combinatorial meanings have been studied in the literature (see $[3,7,23,28]$ ). Recall that the major index, maj $(\pi)$, of a permutation $\pi \in \mathfrak{S}_{n}$ is the sum of all the descents of $\pi$, i.e., $\operatorname{maj}(\pi):=\sum_{\pi(i)>\pi(i+1)} i$. An element $i \in[n]$ is a fixed point of $\pi \in \mathfrak{S}_{n}$ if $\pi(i)=i$ and we denote by fix $(\pi)$ the number of fixed points of $\pi$. Define the $(q, r)$-Eulerian polynomials $A_{n}(t, r, q)$ by the following extension of (1.1):

$$
\sum_{n \geqslant 0} A_{n}(t, r, q) \frac{z^{n}}{(q ; q)_{n}}=\frac{(1-t) e(r z ; q)}{e(t z ; q)-t e(z ; q)}
$$

where $(q ; q)_{n}:=\prod_{i=1}^{n}\left(1-q^{i}\right)$ and $e(z ; q)$ is the $q$-exponential function $\sum_{n \geqslant 0} \frac{z^{n}}{(q ; q)_{n}}$. The following interpretation for $A_{n}(t, r, q)$ was given $[23,25]$ :

$$
A_{n}(t, r, q):=\sum_{\pi \in \mathfrak{S}_{n}} t^{\mathrm{exc} \pi} r^{\mathrm{fix} \pi} q^{(\mathrm{maj}-\mathrm{exc}) \pi} .
$$

These polynomials have attracted the attention of several authors (cf. [10-13, 16-18, 20, $24,26])$.

Let $A_{n}(t, q)=A_{n}(t, 1, q)$. Define the $q$-Eulerian numbers $A_{n, k}(q)$ and the fixed point $q$-Eulerian numbers $A_{n, k}^{(j)}(q)$ :

$$
A_{n}(t, q)=\sum_{k} A_{n, k}(q) t^{k} \quad \text { and } \quad A_{n}(t, r, q)=\sum_{j, k} A_{n, k}^{(j)}(q) r^{j} t^{k}
$$

By (1.3), we have the following interpretations

$$
A_{n, k}(q)=\sum_{\substack{\pi \in \mathfrak{G}_{n} \\ \operatorname{exc} \pi=k}} q^{(\mathrm{maj}-\mathrm{exc}) \pi} \quad \text { and } \quad A_{n, k}^{(j)}(q)=\sum_{\substack{\pi \in \mathfrak{S}_{n} \\ \text { excm }=k \\ \text { fix } \pi=j}} q^{(\mathrm{maj}-\mathrm{exc}) \pi}
$$

Recall that the $q$-binomial coefficients $\left[\begin{array}{l}n \\ k\end{array}\right]_{q}$ are defined by $\left[\begin{array}{l}n \\ k\end{array}\right]_{q}:=\frac{(q ; q)_{n}}{(q ; q)_{n-k}(q ; q)_{k}}$ for $0 \leqslant k \leqslant$ $n$, and $\left[\begin{array}{l}n \\ k\end{array}\right]_{q}=0$ if $k<0$ or $k>n$.

Answering a question of Chung et al. [6], Han et al. [16] found and proved the following symmetrical $q$-Eulerian identity:

$$
\sum_{k \geqslant 1}\left[\begin{array}{c}
a+b \\
k
\end{array}\right]_{q} A_{k, a-1}(q)=\sum_{k \geqslant 1}\left[\begin{array}{c}
a+b \\
k
\end{array}\right]_{q} A_{k, b-1}(q),
$$


where $a, b$ are integers with $a, b \geqslant 1$. Besides a generating function proof using (1.2), a bijective proof of (1.5) was also given in [16]. Recently, through analytical arguments, Chung and Graham [5] derived from (1.2) the following two further symmetrical $q$-Eulerian identities:

$$
\begin{aligned}
\sum_{k \geqslant 1}(-1)^{k}\left[\begin{array}{c}
a+b \\
k
\end{array}\right]_{q} q^{\left(\begin{array}{c}
a+b-k \\
2
\end{array}\right)} A_{k, a}(q) & =\sum_{k \geqslant 1}(-1)^{k}\left[\begin{array}{c}
a+b \\
k
\end{array}\right]_{q} q^{\left(\begin{array}{c}
a+b-k \\
2
\end{array}\right)} A_{k, b}(q), \\
\sum_{k \geqslant 1}\left[\begin{array}{c}
a+b+j+1 \\
k
\end{array}\right]_{q} A_{k, a}^{(j)}(q) & =\sum_{k \geqslant 1}\left[\begin{array}{c}
a+b+j+1 \\
k
\end{array}\right]_{q} A_{k, b}^{(j)}(q),
\end{aligned}
$$

where $a, b, j$ are integers with $a, b \geqslant 1$ and $j \geqslant 0$, and asked for bijective proofs. Our first aim is to provide such proofs using another interpretation of $A_{n}(t, r, q)$ introduced by Foata and Han [11], which was already shown to be successful in the bijective proof of $(1.5)$ in [16].

Next, for $1 \leqslant j \leqslant n$, we shall define the restricted $q$-Eulerian polynomial $B_{n}^{(j)}(t, q)$ by the exponential generating function:

$$
\sum_{n \geqslant j} B_{n}^{(j)}(t, q) \frac{z^{n-1}}{(q ; q)_{n-1}}=\left(\frac{A_{j-1}(t, q)(q z)^{j-1}}{(q ; q)_{j-1}}\right) \frac{e(t z ; q)-t e(t z ; q)}{e(t z ; q)-t e(z ; q)} .
$$

and the restricted $q$-Eulerian number $B_{n, k}^{(j)}(q)$ by $B_{n}^{(j)}(t, q)=\sum_{k} B_{n, k}^{(j)}(q) t^{k}$. We find the following generalized symmetrical identity for the restricted $q$-Eulerian polynomials.

Theorem 1. Let $a, b, j$ be integers with $a, b \geqslant 1$ and $j \geqslant 2$. Then

$$
\sum_{k \geqslant 1}\left[\begin{array}{c}
a+b+1 \\
k-1
\end{array}\right]_{q} B_{k, a}^{(j)}(q)=\sum_{k \geqslant 1}\left[\begin{array}{c}
a+b+1 \\
k-1
\end{array}\right]_{q} B_{k, b}^{(j)}(q) .
$$

When $q=1$, the above identity was proved by Chung and Graham [5], who also asked for a bijective proof. We shall give a bijective proof and an analytical proof of (1.9), the latter leads to a new recurrence formula for $A_{n}(t, r, q)$.

Theorem 2. The (q,r)-Eulerian polynomials satisfy the following recurrence formula:

$$
A_{n+1}(t, r, q)=r A_{n}(t, r, q)+t A_{n}(t, q)+t \sum_{j=1}^{n-1}\left[\begin{array}{l}
n \\
j
\end{array}\right]_{q} q^{j} A_{j}(t, r, q) A_{n-j}(t, q)
$$

for $n \geqslant 1$ and $A_{1}(t, r, q)=r$.

This paper is organized as follows. In section 2, we review some preliminaries about the three-variable statistic (inv, pix, lec) and give the bijective proofs of (1.6) and (1.7). In section 3, we first prove Theorem 2 and then define a new statistic called "rix", which together with descents and admissible inversions (a statistic on permutations which appears in the context of poset topology [23]) gives another interpretation of $A_{n}(t, r, q)$. In section 4 , we give two combinatorial interpretations of $B_{n, k}^{(j)}(q)$ and two proofs of Theorem 1. 


\section{Bijective proofs of (1.6) and (1.7)}

\subsection{Preliminaries}

A word $w=w_{1} w_{2} \ldots w_{m}$ on $\mathbb{N}$ is called a hook if $w_{1}>w_{2}$ and either $m=2$, or $m \geqslant 3$ and $w_{2}<w_{3}<\ldots<w_{m}$. As shown in [14], each permutation $\pi=\pi_{1} \pi_{2} \ldots \pi_{n}$ admits a unique factorization, called its hook factorization, $p \tau_{1} \tau_{2} \cdots \tau_{r}$, where $p$ is an increasing word and each factor $\tau_{1}, \tau_{2}, \ldots, \tau_{k}$ is a hook. To derive the hook factorization of a permutation, one can start from the right and factor out each hook step by step. Denote by $\operatorname{inv}(w)$ the numbers of inversions of a word $w=w_{1} w_{2} \ldots w_{m}$, i.e., the number of pairs $\left(w_{i}, w_{j}\right)$ such that $i<j$ and $w_{i}>w_{j}$. Then we define

$$
\operatorname{lec}(\pi):=\sum_{1 \leqslant i \leqslant k} \operatorname{inv}\left(\tau_{i}\right) \text { and } \operatorname{pix}(\pi)=|p|:=\text { length of the factor } p .
$$

For example, the hook factorization of $\pi=134141225111586713910$ is

$$
13414|12251115| 867 \mid 13910 .
$$

Hence $p=13414, \tau_{1}=12251115, \tau_{2}=867, \tau_{3}=13910, \operatorname{pix}(\pi)=4$ and

$$
\operatorname{lec}(\pi)=\operatorname{inv}(12251115)+\operatorname{inv}(867)+\operatorname{inv}(13910)=7 .
$$

Let $\mathcal{A}_{0}, \mathcal{A}_{1}, \ldots, \mathcal{A}_{r}$ be a series of sets on $\mathbb{N}$. Denote by $\operatorname{inv}\left(\mathcal{A}_{0}, \mathcal{A}_{1}, \ldots, \mathcal{A}_{r}\right)$ the number of pairs $(k, l)$ such that $k \in \mathcal{A}_{i}, l \in \mathcal{A}_{j}, k>l$ and $i<j$. We usually write $\operatorname{cont}(\mathcal{A})$ the set of all letters in a word $\mathcal{A}$. So we have (inv $-\operatorname{lec}) \pi=\operatorname{inv}\left(\operatorname{cont}(p), \operatorname{cont}\left(\tau_{1}\right), \ldots, \operatorname{cont}\left(\tau_{r}\right)\right)$ if $p \tau_{1} \tau_{2} \cdots \tau_{r}$ is the hook factorization of $\pi$.

From Foata and Han [11, Theorem 1.4], we derive the following combinatorial interpretations of the $(q, r)$-Eulerian polynomials

$$
A_{n}(t, r, q)=\sum_{\pi \in \mathfrak{G}_{n}} t^{\mathrm{lec} \pi} r^{\mathrm{pix} \pi} q^{(\mathrm{inv}-\mathrm{lec}) \pi} .
$$

Therefore

$$
A_{n, k}(q)=\sum_{\substack{\pi \in \mathfrak{G}_{n} \\ \operatorname{lec} \pi=k}} q^{(\mathrm{inv}-\mathrm{lec}) \pi} \quad \text { and } \quad A_{n, k}^{(j)}(q)=\sum_{\substack{\pi \in \mathfrak{G}_{n} \\ \text { locink } \\ \operatorname{pix} \times=j}} q^{(\mathrm{inv}-\mathrm{lec}) \pi} .
$$

It is known [27, Proposition 1.3.17] that the $q$-binomial coefficient has the interpretation

$$
\left[\begin{array}{l}
n \\
k
\end{array}\right]_{q}=\sum_{(\mathcal{A}, \mathcal{B})} q^{\operatorname{inv}(\mathcal{A}, \mathcal{B})}
$$

where the sum is over all ordered partitions $(\mathcal{A}, \mathcal{B})$ of $[n]$ such that $|\mathcal{A}|=k$. We will give bijective proofs of (1.6) and (1.7) using the interpretations in (2.2) and (2.3). 
Remark 1. In [11], a bijection on $\mathfrak{S}_{n}$ that carries the triplet (fix, exc, maj) to (pix, lec, inv) was constructed without being specified. This bijection consists of two steps. The first step (see [11, section 6]) uses the word analogue of Kim-Zeng's decomposition [19] and an updated version of Gessel-Reutenauer standardization [15] to construct a bijection on $\mathfrak{S}_{n}$ that transforms the triplet (fix, exc, maj) to (pix, lec, imaj), where imaj $(\pi):=\operatorname{maj}\left(\pi^{-1}\right)$ for each permutation $\pi$. The second step (see [11, section 7]) uses Foata's second fundamental transformation [8] to carry the triplet (pix, lec,imaj) to (pix, lec, inv). In view of this bijection, one can construct bijective proofs of (1.5), (1.6) and (1.7) using the original interpretations in (1.4), through the bijective proof of (1.5) in [16] and our bijective proofs.

To construct our bijective proofs, we need two elementary transformations from [16] that we recall now. Let $\tau$ be a hook with $\operatorname{inv}(\tau)=k$ and $\operatorname{cont}(\tau)=\left\{x_{1}, \ldots, x_{m}\right\}$, where $x_{1}<\ldots<x_{m}$. Define

$$
d(\tau)=x_{m-k+1} x_{1} \ldots x_{m-k} x_{m-k+2} \ldots x_{m} .
$$

Clearly, $d(\tau)$ is the unique hook with $\operatorname{cont}(d(\tau))=\operatorname{cont}(\tau)$ and satisfying

$$
\operatorname{inv}(d(\tau))=m-k=|\operatorname{cont}(\tau)|-\operatorname{inv}(\tau) .
$$

Let $\tau$ be a hook or an increasing word with $\operatorname{inv}(\tau)=k$ and $\operatorname{cont}(\tau)=\left\{x_{1}, \ldots, x_{m}\right\}$, where $x_{1}<\ldots<x_{m}$. Define

$$
d^{\prime}(\tau)=x_{m-k} x_{1} \ldots x_{m-k-1} x_{m-k+1} \ldots x_{m} .
$$

It is not difficult to see that, $d^{\prime}(\tau)$ is the unique hook (when $k<m-1$ ) or increasing word $($ when $k=m-1)$ with $\operatorname{cont}(d(\tau))=\operatorname{cont}(\tau)$ and satisfying

$$
\operatorname{inv}(d(\tau))=m-k-1=|\operatorname{cont}(\tau)|-1-\operatorname{inv}(\tau) .
$$

\subsection{Bijective proof of (1.6)}

Let $\mathfrak{S}_{n}(k)=\left\{\pi \in \mathfrak{S}_{n}: \operatorname{pix}(\pi)=k\right\}$ and $\mathcal{D}_{n}=\mathfrak{S}_{n}(0)$. We first notice that the left-hand side of (1.6) has the following interpretation:

$$
\sum_{\substack{\pi \in \mathcal{D}_{n} \\
\text { lec } \pi=a}} q^{(\mathrm{inv}-\mathrm{lec}) \pi}=\sum_{k \geqslant 1}(-1)^{n-k}\left[\begin{array}{l}
n \\
k
\end{array}\right]_{q} q^{\left(\frac{n-k}{2}\right)} A_{k, a}(q) .
$$


This interpretation follows immediately from [25, Corollary 4.4] and (2.1). One can also give a direct combinatorial proof similarly as in [29]. Actually, by (2.2) and (2.3) we have

$$
\begin{aligned}
A_{n, a}(q) & =\sum_{\substack{\pi \in \mathfrak{G}_{n} \\
\text { lec } \pi=a}} q^{(\mathrm{inv}-\mathrm{lec}) \pi} \\
& =\sum_{k} \sum_{\substack{\pi \in \mathfrak{S}_{n}(k) \\
\text { lec } \pi=a}} q^{\operatorname{inv}\left(\operatorname{cont}(p), \operatorname{cont}\left(\tau_{1} \ldots \tau_{r}\right)\right)+\operatorname{inv}\left(\operatorname{cont}\left(\tau_{1}\right), \operatorname{cont}\left(\tau_{2}\right), \ldots, \operatorname{cont}\left(\tau_{r}\right)\right)} \\
& =\sum_{k} \sum_{\substack{\mathcal{A} \in[n] \\
|\mathcal{A}|=k}} q^{\operatorname{inv}(\mathcal{A},[n] \backslash \mathcal{A})} \sum_{\substack{\pi \in \mathcal{D}_{n-k}-k \\
\text { lec } \pi=a}} q^{(\operatorname{inv}-\operatorname{lec}) \pi} \\
& =\sum_{k}\left[\begin{array}{l}
n \\
k
\end{array} \sum_{\substack{\pi \in \mathcal{D}_{k} \\
\text { lec } \pi=a}} q^{(\mathrm{inv}-\operatorname{lec}) \pi} .\right.
\end{aligned}
$$

Applying Gaussian inversion (or $q$-binomial inversion) to the above identity gives (2.6).

Now, by (2.6), the symmetrical identity (1.6) is equivalent to the $j=0$ case of the following Lemma.

Lemma 3. For $0 \leqslant j \leqslant n$, there is an involution $\mathbf{v} \mapsto \mathbf{u}$ on $\mathfrak{S}_{n}(j)$ satisfying

$$
\operatorname{lec}(\mathbf{u})=n-j-\operatorname{lec}(\mathbf{v}) \quad \text { and }(i n v-l e c) \mathbf{u}=(i n v-l e c) \mathbf{v} .
$$

Proof. Let $\mathbf{v}=p \tau_{1} \tau_{2} \ldots \tau_{r}$ be the hook factorization of $\mathbf{v} \in \mathfrak{S}_{n}(j)$, where $p$ is an increasing word and each factor $\tau_{1}, \tau_{2}, \ldots, \tau_{r}$ is a hook. We define $\mathbf{u}=p d\left(\tau_{1}\right) \ldots d\left(\tau_{r}\right)$, where $d$ is defined in (2.4). It is easy to check that this mapping is an involution on $\mathfrak{S}_{n}(j)$ with the desired properties.

By (2.2), Lemma 3 gives a simple bijective proof of the following known $[5,25]$ symmetric property of the fixed point $q$-Eulerian numbers.

Corollary 4. For $n, k, j \geqslant 0$,

$$
A_{n, k}^{(j)}(q)=A_{n, n-j-k}^{(j)}(q)
$$

\subsection{Bijective proof of (1.7)}

Recall [16] that, for a fixed positive integer $n$, a two-pix-permutation of $[n]$ is a sequence of words

$$
\mathbf{v}=\left(p_{1}, \tau_{1}, \tau_{2}, \ldots, \tau_{r-1}, \tau_{r}, p_{2}\right)
$$

satisfying the following conditions:

(C1) $p_{1}$ and $p_{2}$ are two increasing words, possibly empty;

(C2) $\tau_{1}, \ldots, \tau_{r}$ are hooks for some positive integer $r$;

(C3) The concatenation $p_{1} \tau_{1} \tau_{2} \ldots \tau_{r-1} \tau_{r} p_{2}$ of all components of $\mathbf{v}$ is a permutation of $[n]$. 
We also extend the two statistics to the two-pix-permutations by

$$
\operatorname{lec}(\mathbf{v})=\sum_{1 \leqslant i \leqslant r} \operatorname{inv}\left(\tau_{i}\right) \quad \text { and } \quad \operatorname{inv}(\mathbf{v})=\operatorname{inv}\left(p_{1} \tau_{1} \tau_{2} \ldots \tau_{r-1} \tau_{r} p_{2}\right)
$$

It follows that

$$
(\operatorname{inv}-\operatorname{lec}) \mathbf{v}=\operatorname{inv}\left(\operatorname{cont}\left(p_{1}\right), \operatorname{cont}\left(\tau_{1}\right), \operatorname{cont}\left(\tau_{2}\right), \ldots, \operatorname{cont}\left(\tau_{r}\right), \operatorname{cont}\left(p_{2}\right)\right)
$$

Let $\mathcal{W}_{n}(j)$ denote the set of all two-pix-permutations with $\left|p_{1}\right|=j$.

Lemma 5. Let $a, j$ be fixed nonnegative integers. Then

$$
\sum_{\substack{\mathbf{v} \in \mathcal{W}_{n}(j) \\
l e c \mathbf{v}=a}} q^{(i n v-l e c) \mathbf{v}}=\sum_{k \geqslant 1}\left[\begin{array}{l}
n \\
k
\end{array}\right]_{q} A_{k, a}^{(j)}(q) .
$$

Proof. By the hook factorization, the two-pix-permutation in (2.8) is in bijection with the pair $\left(\sigma, p_{2}\right)$, where $\sigma=p_{1} \tau_{1} \tau_{2} \ldots \tau_{r-1} \tau_{r}$ is a permutation on $[n] \backslash \operatorname{cont}\left(p_{2}\right)$ and $p_{2}$ is an increasing word. Thus, by (2.2), (2.3) and (2.9), the generating function of all two-pixpermutations $\mathbf{v}$ of $[n]$ with $\left|p_{1}\right|=j$ such that $\operatorname{lec}(\mathbf{v})=a$ and $\left|p_{2}\right|=n-k$ with respect to the weight $q^{(\mathrm{inv}-\mathrm{lec}) \mathbf{v}}$ is $\left[\begin{array}{c}n \\ n-k\end{array}\right]_{q} A_{k, a}^{(j)}(q)$.

Lemma 6. Let $j$ be a fixed nonnegative integer. Then there is an involution $\mathbf{v} \mapsto \mathbf{u}$ on $\mathcal{W}_{n}(j)$ satisfying

$$
\operatorname{lec}(\mathbf{v})=n-j-1-\operatorname{lec}(\mathbf{u}), \quad \text { and } \quad(i n v-l e c) \mathbf{v}=(i n v-l e c) \mathbf{u} .
$$

Proof. We give an explicit construction of the bijection using the involutions $d$ and $d^{\prime}$ defined in (2.4) and (2.5).

Let $\mathbf{v}=\left(p_{1}, \tau_{1}, \tau_{2}, \ldots, \tau_{r-1}, \tau_{r}, p_{2}\right)$ be a two-pix-permutation of $[n]$ with $\left|p_{1}\right|=j$. If $p_{2} \neq \emptyset$, then

$$
\mathbf{u}=\left(p_{1}, d\left(\tau_{1}\right), d\left(\tau_{2}\right), \ldots, d\left(\tau_{r-1}\right), d\left(\tau_{r}\right), d^{\prime}\left(p_{2}\right)\right)
$$

otherwise,

$$
\mathbf{u}=\left(p_{1}, d\left(\tau_{1}\right), d\left(\tau_{2}\right), \ldots, d\left(\tau_{r-1}\right), d^{\prime}\left(\tau_{r}\right)\right)
$$

As $d$ and $d^{\prime}$ are involutions, this mapping is an involution on $\mathcal{W}_{n}(j)$.

Since we have $\operatorname{lec}\left(d\left(\tau_{i}\right)\right)=\left|\operatorname{cont}\left(\tau_{i}\right)\right|-\operatorname{lec}\left(\tau_{i}\right)$ for $1 \leqslant i \leqslant r$ and $\operatorname{lec}\left(d^{\prime}\left(p_{2}\right)\right)=\left|\operatorname{cont}\left(p_{2}\right)\right|-$ 1 in the case $p_{2} \neq \emptyset$, it follows that $\operatorname{lec}(\mathbf{u})=\sum_{i=1}^{r}\left|\operatorname{cont}\left(\tau_{i}\right)\right|+\left|\operatorname{cont}\left(p_{2}\right)\right|-1-\operatorname{lec}(\mathbf{v})=$ $n-j-1-\operatorname{lec}(\mathbf{v})$. The above identity is also valid when $p_{2}=\emptyset$.

Finally it follows from $(2.9)$ that $(i n v-l e c) \mathbf{u}=($ inv $-l e c) \mathbf{v}$. This finishes the proof of the lemma.

Combining Lemmas 5 and 6 we obtain a bijective proof of (1.7). 


\section{A new recurrence formula for the $(q, r)$-Eulerian polynomials}

The Eulerian differential operator $\delta_{x}$ used below is defined by

$$
\delta_{x}(f(x)):=\frac{f(x)-f(q x)}{x}
$$

for any $f(x) \in \mathbb{Q}[q][[x]]$ in the ring of formal power series in $x$ over $\mathbb{Q}[q]$ (instead of the traditional $(f(x)-f(q x)) /((1-q) x)$, see $[1,4])$. We need the following elementary properties of $\delta_{x}$.

Lemma 7. For any $f(x), g(x) \in \mathbb{Q}[q][[x]]$,

$$
\delta_{x}(f(x) g(x))=f(q x) \delta(g(x))+\delta(f(x)) g(x)
$$

and

$$
\delta_{x}\left(\frac{1}{f(x)}\right)=\frac{-\delta_{x}(f(x))}{f(q x) f(x)} \quad(f(x) \neq 0) .
$$

Proof of Theorem 2. It is not difficult to show that, for any variable $a$

$$
\delta_{z}(e(a z ; q))=a e(a z ; q)
$$

Now, applying $\delta_{z}$ to both sides of (1.2) and using the above property and Lemma 7, we obtain

$$
\begin{aligned}
& \sum_{n \geqslant 0} A_{n+1}(t, r, q) \frac{z^{n}}{(q ; q)_{n}} \\
& =\delta_{z}\left(\frac{(1-t) e(r z ; q)}{e(t z ; q)-t e(z ; q)}\right) \\
& =\delta_{z}((1-t) e(r z ; q))(e(t z ; q)-t e(z ; q))^{-1}+\delta_{z}\left((e(t z ; q)-t e(z ; q))^{-1}\right)(1-t) e(r z q ; q) \\
& =\frac{r(1-t) e(r z ; q)}{e(t z ; q)-t e(z ; q)}+\frac{(1-t) e(r z q ; q)(t e(z ; q)-t e(t z ; q))}{(e(t q z ; q)-t e(q z ; q))(e(t z ; q)-t e(z ; q))} \\
& =r \sum_{n \geqslant 0} A_{n}(t, r, q) \frac{z^{n}}{(q ; q)_{n}}+t\left(\sum_{n \geqslant 0} A_{n}(t, r, q) \frac{(q z)^{n}}{(q ; q)_{n}}\right)\left(\sum_{n \geqslant 1} A_{n}(t, q) \frac{z^{n}}{(q ; q)_{n}}\right)
\end{aligned}
$$

Taking the coefficient of $\frac{z^{n}}{(q ; q)_{n}}$ in both sides of the above equality, we get (1.10).

Remark 2. A different recurrence formula for $A_{n}(t, r, q)$ was obtained in [25, Corollary 4.3]. Eq. (1.10) is similar to two recurrence formulas in the literature: one for the (inv, des)- $q$-Eulerian polynomials in [21, Corollary 2.22] (see also [4]) and the other one for the (maj, des)- $q$-Eulerian polynomials in [21, Corollary 3.6]. 
We shall give another interpretation of $A_{n}(t, r, q)$ in the following.

Let $\pi \in \mathfrak{S}_{n}$. Recall that an inversion of $\pi$ is a pair $(\pi(i), \pi(j))$ such that $1 \leqslant i<j \leqslant n$ and $\pi(i)>\pi(j)$. An admissible inversion of $\pi$ is an inversion $(\pi(i), \pi(j))$ that satisfies either

- $1<i$ and $\pi(i-1)<\pi(i)$ or

- there is some $l$ such that $i<l<j$ and $\pi(i)<\pi(l)$.

We write ai $(\pi)$ the number of admissible inversions of $\pi$. Define the statistic $\operatorname{aid}(\pi):=$ ai $(\pi)+\operatorname{des}(\pi)$. For example, if $\pi=42153$ then there are 5 inversions, but only $(4,3)$ and $(5,3)$ are admissible. So $\operatorname{inv}(\pi)=5$, ai $(\pi)=2$ and $\operatorname{aid}(\pi)=2+3=5$. The statistics ai and aid were first studied by Shareshian and Wachs [23] in the context of Poset Topology. Here we follow the definitions in [20]. The curious result that the pairs (aid, des) and (maj, exc) are equidistributed on $\mathfrak{S}_{n}$ was proved in [20] using techniques from poset topology.

Let $\mathcal{W}$ be the set of all the words on $\mathbb{N}$. We define a new statistic, denoted by "rix", on $\mathcal{W}$ recursively. Let $W=w_{1} w_{2} \cdots w_{n}$ be a word in $\mathcal{W}$ and $w_{i}$ be the rightmost maximum element of $W$. We define $\operatorname{rix}(W)$ by (with convention that $\operatorname{rix}(\emptyset)=0$ )

$$
\operatorname{rix}(W):= \begin{cases}0, & \text { if } i=1 \neq n, \\ 1+\operatorname{rix}\left(w_{1} \cdots w_{n-1}\right), & \text { if } i=n, \\ \operatorname{rix}\left(w_{i+1} w_{i+2} \cdots w_{n}\right), & \text { if } 1<i<n .\end{cases}
$$

For example, we have $\operatorname{rix}(1524335)=1+\operatorname{rix}(152433)=1+\operatorname{rix}(2433)=1+\operatorname{rix}(33)=$ $2+\operatorname{rix}(3)=3$. As every permutation can be viewed as a word on $\mathbb{N}$, this statistic is welldefined on permutations.

We write $\mathfrak{S}_{n}^{(j)}$ the set of permutations $\pi \in \mathfrak{S}_{n}$ with $\pi(j)=n$. For $n \geqslant 1$ and $1 \leqslant j \leqslant n$, we define $B_{n}(t, r, q):=\sum_{\pi \in \mathfrak{S}_{n}} t^{\operatorname{des} \pi} r^{\mathrm{rix} \pi} q^{\mathrm{ai} \pi}$ and its restricted version by

$$
B_{n}^{(j)}(t, r, q):=\sum_{\pi \in \mathfrak{S}_{n}^{(j)}} t^{\mathrm{des} \pi} r^{\mathrm{rix} \pi} q^{\mathrm{ai} \pi} .
$$

We should note here that the restricted $q$-Eulerian polynomial $B_{n}^{(j)}(t, q)$ is not equal to $B_{n}^{(j)}(t, 1, q)$ but some modification of it, as will be shown in the next section.

Theorem 8. We have the following interpretation for $(q, r)$-Eulerian polynomials:

$$
A_{n}(t, r, q)=\sum_{\pi \in \mathfrak{S}_{n}} t^{d e s \pi} r^{r i x \pi} q^{a i \pi} .
$$

Proof. We will show that $B_{n}(t, r, q)$ satisfies the same recurrence formula and initial condition as $A_{n}(t, r, q)$. For $n \geqslant 1$, it is clear from the definition of $B_{n}(t, r, q)$ that

$$
B_{n+1}(t, r, q)=\sum_{1 \leqslant j \leqslant n+1} B_{n+1}^{(j)}(t, r, q) .
$$


It is easy to see that

$$
B_{n+1}^{(1)}(t, r, q)=t B_{n}(t, 1, q) \quad \text { and } \quad B_{n+1}^{(n+1)}(t, r, q)=r B_{n}(t, r, q) .
$$

We then consider $B_{n+1}^{(j)}(t, r, q)$ for the case of $1<j<n+1$.

For a set $X$, we denote by $\left(\begin{array}{l}X \\ m\end{array}\right)$ the $m$-element subsets of $X$ and $\mathfrak{S}_{X}$ the set of permutations of $X$. Let $\mathcal{W}(n, j)$ be the set of all triples $\left(W, \pi_{1}, \pi_{2}\right)$ such that $W \in\left(\begin{array}{c}{[n]} \\ j\end{array}\right)$ and $\pi_{1} \in \mathfrak{S}_{W}, \pi_{2} \in \mathfrak{S}_{[n] \backslash W}$. It is not difficult to see that the mapping $\pi \mapsto\left(W, \pi_{1}, \pi_{2}\right)$ defined by

- $W=\{\pi(i): 1 \leqslant i \leqslant j-1\}$,

- $\pi_{1}=\pi(1) \pi(2) \cdots \pi(j-1)$ and $\pi_{2}=\pi(j+1) \pi(j+2) \cdots \pi(n)$

is a bijection between $\mathfrak{S}_{n}^{(j)}$ and $\mathcal{W}(n-1, j-1)$ and satisfies

$$
\operatorname{des}(\pi)=\operatorname{des}\left(\pi_{1}\right)+\operatorname{des}\left(\pi_{2}\right)+1, \quad \operatorname{rix}(\pi)=\operatorname{rix}\left(\pi_{2}\right)
$$

and

$$
\operatorname{ai}(\pi)=\operatorname{ai}\left(\pi_{1}\right)+\operatorname{ai}\left(\pi_{2}\right)+\operatorname{inv}(W,[n-1] \backslash W)+n-j .
$$

Thus, for $1<j<n+1$, we have

$$
\begin{aligned}
B_{n+1}^{(j)}(t, r, q) & =\sum_{\pi \in \mathfrak{S}_{n+1}^{(j)}} t^{\mathrm{des} \pi} r^{\mathrm{rix} \pi} q^{\mathrm{ai} \pi} \\
& =t q^{n+1-j} \sum_{\left(W, \pi_{1}, \pi_{2}\right) \in \mathcal{W}(n, j-1)} q^{\operatorname{inv}(W,[n] \backslash W)} q^{\mathrm{ai}\left(\pi_{1}\right)} t^{\operatorname{des}\left(\pi_{1}\right)} r^{\mathrm{rix}\left(\pi_{2}\right)} q^{\mathrm{ai}\left(\pi_{2}\right)} t^{\operatorname{des}\left(\pi_{2}\right)} \\
& =t q^{n+1-j} \sum_{W \in\left(\begin{array}{l}
{[n]} \\
j-1
\end{array}\right)} q^{\operatorname{inv}(W,[n] \backslash W)} \sum_{\pi \in \mathfrak{S}_{W}} q^{\mathrm{ai}\left(\pi_{1}\right)} t^{\operatorname{des}\left(\pi_{1}\right)} \sum_{\pi_{2} \in \mathfrak{S}_{[n] \backslash W}} r^{\operatorname{rix}\left(\pi_{2}\right)} q^{\mathrm{ai}\left(\pi_{2}\right)} t^{\operatorname{des}\left(\pi_{2}\right)} \\
& =t q^{n+1-j}\left[\begin{array}{c}
n \\
j-1
\end{array}\right]_{q} B_{j-1}(t, 1, q) B_{n+1-j}(t, r, q),
\end{aligned}
$$

where we apply (2.3) to the last equality. Substituting (3.4) and (3.5) into (3.3) we obtain

$$
B_{n+1}(t, r, q)=r B_{n}(t, r, q)+t B_{n}(t, 1, q)+t \sum_{j=1}^{n-1}\left[\begin{array}{l}
n \\
j
\end{array}\right]_{q} q^{j} B_{j}(t, r, q) B_{n-j}(t, 1, q) .
$$

By Theorem 2, $B_{n}(t, r, q)$ and $A_{n}(t, r, q)$ satisfy the same recurrence formula and initial condition, thus $B_{n}(t, r, q)=A_{n}(t, r, q)$. This finishes the proof of the theorem.

Corollary 9. The three triplets (rix, des, aid), (fix, exc, maj) and (pix, lec, inv) are equidistributed on $\mathfrak{S}_{n}$.

Remark 3. At the Permutation Patterns 2012 conference, Alexander Burstein [2] gave a direct bijection on $\mathfrak{S}_{n}$ that transforms the triple (rix, des, aid) to (pix, lec, inv). The new statistic "rix" was introduced independently therein under the name "aix". Actually, the definitions of both are slightly different, but they are the same up to an easy transformation. It would be very interesting to find a similar bijective proof of the equidistribution of (rix, des, aid) and (fix, exc, maj). See also Remark 1. 


\section{A symmetrical identity for restricted $q$-Eulerian polynomials}

\subsection{An interpretation of $B_{n, k}^{(j)}(q)$ and a proof of Theorem 1}

It follows from (1.2) and (1.8) that $B_{1,0}^{(1)}(q)=1$ and $B_{n, k}^{(1)}(q)=A_{n-1, k-1}(q)$ for $k \geqslant 1$. For $j \geqslant 2$, we have the following interpretation for $B_{n, k}^{(j)}(q)$.

Lemma 10. For $2 \leqslant j \leqslant n, B_{n, k}^{(j)}(q)=\sum_{\substack{\pi \in \mathfrak{G}_{n}^{(j)} \\ \operatorname{des}(\pi)=k}} q^{a i(\pi)+2 j-n-1}$.

Proof. When $j \geqslant 2$, by the recurrence relation (3.5), one can compute without difficulty that the exponential generating function $\sum_{n \geqslant j} q^{2 j-n-1} B_{n}^{(j)}(t, 1, q) \frac{z^{n-1}}{(q ; q)_{n-1}}$ is exactly the right side of (1.8) using (1.2) and (3.2), which would finish the proof of the lemma.

Originally, the restricted Eulerian number $B_{n, k}^{(j)}$ in [5] was defined to be the number of permutations $\pi \in \mathfrak{S}_{n}$ with $\operatorname{des}(\pi)=k$ and $\pi(j)=n$. According to the above lemma, $B_{n, k}^{(j)}(q)$ is really a $q$-analogue of $B_{n, k}^{(j)}$. This justifies the names restricted $q$-Eulerian number and restricted $q$-Eulerian polynomials.

Lemma 11. For $1<j<n$, we have

$$
B_{n, k}^{(j)}(q)=B_{n, n-1-k}^{(j)}(q)
$$

Proof. We first construct an involution $f: \pi \mapsto \pi^{\prime}$ on $\mathfrak{S}_{n}$ satisfying

$$
\operatorname{ai}(\pi)=\operatorname{ai}\left(\pi^{\prime}\right) \quad \text { and } \quad \operatorname{des}(\pi)=n-1-\operatorname{des}\left(\pi^{\prime}\right) .
$$

For $n=1$, define $f(\mathrm{id})=\mathrm{id}$. For $n \geqslant 2$, suppose that $\pi=\pi_{1} \cdots \pi_{n}$ is a permutation of $\left\{\pi_{1}, \cdots, \pi_{n}\right\}$ and $\pi_{j}$ is the maximum element in $\left\{\pi_{1}, \cdots, \pi_{n}\right\}$. We construct $f$ recursively as follows

$$
f(\pi)= \begin{cases}f\left(\pi_{2} \pi_{3} \cdots \pi_{n}\right) \pi_{1}, & \text { if } j=1 \\ \pi_{n} f\left(\pi_{1} \pi_{2} \cdots \pi_{n-1}\right), & \text { if } j=n \\ f\left(\pi_{1} \pi_{2} \cdots \pi_{j-1}\right) \pi_{j} f\left(\pi_{j+1} \pi_{j+2} \cdots \pi_{n}\right), & \text { otherwise }\end{cases}
$$

For example, if $\pi=3257641$, then

$$
f(\pi)=f(325) 7 f(641)=5 f(32) 7 f(41) 6=5237146 .
$$

Clearly, $\operatorname{ai}(\pi)=7=\operatorname{ai}\left(\pi^{\prime}\right)$ and $\operatorname{des}(\pi)=4=7-1-\operatorname{des}\left(\pi^{\prime}\right)$. It is not difficult to see that $f$ is an involution. We can show that $f$ satisfies (4.1) by induction on $n$, which is routine and left to the reader.

For each $\pi=\pi_{1} \cdots \pi_{j-1} n \pi_{j+1} \cdots \pi_{n}$ in $\mathfrak{S}_{n}^{(j)}$, we then define

$$
g(\pi)=f\left(\pi_{1} \cdots \pi_{j-1}\right) n f\left(\pi_{j+1} \cdots \pi_{n}\right) .
$$

As $f$ is an involution, $g$ is an involution on $\mathfrak{S}_{n}^{(j)}$. It follows from $(4.1)$ that ai $(g(\pi))=\operatorname{ai}(\pi)$ and $\operatorname{des}(\pi)=n-1-\operatorname{des}(g(\pi))$, which completes the proof in view of Lemma 10 . 
Remark 4. A bijective proof of Lemma 11 when $q=1$ was given in [5]. But their bijection does not preserve the admissible inversions. Supposing that $\pi=\pi_{1} \cdots \pi_{n}$ is a permutation of $\left\{\pi_{1}, \cdots, \pi_{n}\right\}$ and $\pi_{j}$ is the maximum element in $\left\{\pi_{1}, \cdots, \pi_{n}\right\}$, we modify $f$ defined above to $f^{\prime}$ as follows:

$$
f^{\prime}(\pi)= \begin{cases}f^{\prime}\left(\pi_{2} \pi_{3} \cdots \pi_{n}\right) \pi_{1}, & \text { if } j=1, \\ \pi, & \text { if } j=n, \\ f^{\prime}\left(\pi_{1} \pi_{2} \cdots \pi_{j-1}\right) \pi_{j} f^{\prime}\left(\pi_{j+1} \pi_{j+2} \cdots \pi_{n}\right), & \text { otherwise. }\end{cases}
$$

The reader is invited to check that $f^{\prime}$ would provide another bijective proof of Corollary 4 using (des, rix, ai).

Now we are in position to give a generating function proof of Theorem 1.

Proof of Theorem 1. We start with the generating function given in (1.8). Multiplying both sides by $e(t z ; q)-t e(z ; q)$, we obtain

$$
\sum_{n, k} B_{n, k}^{(j)}(q) t^{k} \frac{z^{n-1}}{(q ; q)_{n-1}}(e(t z ; q)-t e(z ; q))=\frac{(q z)^{j-1} A_{j-1}(t, q)}{(q ; q)_{j-1}}(e(t z ; q)-t e(t z ; q)) .
$$

Expanding the exponential functions, we have

$$
\begin{aligned}
\sum_{n, k, i} B_{n, k}^{(j)}(q) \frac{t^{k+i} z^{n+i-1}}{(q ; q)_{i}(q ; q)_{n-1}} & -\sum_{n, k, i} B_{n, k}^{(j)}(q) \frac{t^{k+1} z^{n+i-1}}{(q ; q)_{i}(q ; q)_{n-1}} \\
& =\frac{(q z)^{j-1} A_{j-1}(t, q)}{(q ; q)_{j-1}} \sum_{n \geqslant 0} \frac{(1-t) t^{n} z^{n}}{(q ; q)_{n}} .
\end{aligned}
$$

Identifying the coefficient of $t^{l} z^{m-1}$ gives

$$
\begin{aligned}
\sum_{k} \frac{B_{m+k-l, k}^{(j)}(q)}{(q ; q)_{l-k}(q ; q)_{m+k-l-1}} & -\sum_{i} \frac{B_{m-i, l-1}^{(j)}(q)}{(q ; q)_{i}(q ; q)_{m-i-1}} \\
& =\frac{q^{j-1}\left(A_{j-1, l+j-m}(q)-A_{j-1, l+j-m-1}(q)\right)}{(q ; q)_{j-1}(q ; q)_{m-j}} .
\end{aligned}
$$

Multiplying both sides by $(q ; q)_{m-1}$, we get

$$
\begin{aligned}
\sum_{k} B_{m+k-l, k}^{(j)}(q)\left[\begin{array}{c}
m-1 \\
l-k
\end{array}\right]_{q} & -\sum_{i} B_{m-i, l-1}^{(j)}(q)\left[\begin{array}{c}
m-1 \\
i
\end{array}\right]_{q} \\
& =\left(A_{j-1, l+j-m}(q)-A_{j-1, l+j-m-1}(q)\right) q^{j-1}\left[\begin{array}{c}
m-1 \\
j-1
\end{array}\right]_{q} .
\end{aligned}
$$


Changing the variables of the two summations on the left side gives

$$
\begin{aligned}
\sum_{k} B_{k, k+l-m}^{(j)}(q)\left[\begin{array}{c}
m-1 \\
k-1
\end{array}\right]_{q} & -\sum_{k} B_{k, l-1}^{(j)}(q)\left[\begin{array}{c}
m-1 \\
k-1
\end{array}\right]_{q} \\
& =\left(A_{j-1, l+j-m}(q)-A_{j-1, l+j-m-1}(q)\right) q^{j-1}\left[\begin{array}{c}
m-1 \\
j-1
\end{array}\right]_{q}
\end{aligned}
$$

We apply the symmetric property in Lemma 11 to the first summation on the left side of (4.2) and we have

$$
\begin{aligned}
& \sum_{k} B_{k, k+l-m}^{(j)}(q)\left[\begin{array}{c}
m-1 \\
k-1
\end{array}\right]_{q} \\
& =B_{j, j+l-m}^{(j)}(q)\left[\begin{array}{c}
m-1 \\
j-1
\end{array}\right]_{q}+\sum_{k \neq j} B_{k, m-1-l}^{(j)}(q)\left[\begin{array}{c}
m-1 \\
k-1
\end{array}\right]_{q} .
\end{aligned}
$$

It follows from Lemma 10 and Theorem 8 that

$$
B_{n, k}^{(n)}(q)=\sum_{\substack{\pi \in \mathfrak{S}_{n}^{(n)} \\ \operatorname{des}(\pi)=k}} q^{\mathrm{ai}(\pi)+n-1}=q^{n-1} A_{n-1, k}(q) .
$$

Using the symmetric property of $A_{n, k}(q)$, that is $A_{n, k}(q)=A_{n, n-1-k}(q)$, and the above property, the right side of (4.2) can be treated as follows:

$$
\begin{aligned}
& \left(A_{j-1, l+j-m}(q)-A_{j-1, l+j-m-1}(q)\right) q^{j-1}\left[\begin{array}{c}
m-1 \\
j-1
\end{array}\right]_{q} \\
& =B_{j, j+l-m}^{(j)}(q)\left[\begin{array}{c}
m-1 \\
j-1
\end{array}\right]_{q}-A_{j-1, m-1-l}(q) q^{j-1}\left[\begin{array}{c}
m-1 \\
j-1
\end{array}\right]_{q} \\
& =B_{j, j+l-m}^{(j)}(q)\left[\begin{array}{c}
m-1 \\
j-1
\end{array}\right]_{q}-B_{j, m-1-l}^{(j)}(q)\left[\begin{array}{c}
m-1 \\
j-1
\end{array}\right]_{q} .
\end{aligned}
$$

Now we substitute (4.3), (4.4) into (4.2) and obtain

$$
\sum_{k} B_{k, m-1-l}^{(j)}(q)\left[\begin{array}{c}
m-1 \\
k-1
\end{array}\right]_{q}=\sum_{k} B_{k, l-1}^{(j)}(q)\left[\begin{array}{c}
m-1 \\
k-1
\end{array}\right]_{q}
$$

which becomes (1.9) after setting $m=a+b+2$ and $l-1=b$.

Remark 5. The only case that is left out in Theorem 1 is the case of $j=1$. However, as $B_{n, k}^{(1)}(q)=A_{n-1, k-1}(q)$, the corresponding symmetrical identity for this case is (1.5). 


\subsection{Another interpretation of $B_{n, k}^{(j)}(q)$ and a bijective proof of Theorem 1}

Let

$$
\overline{\mathfrak{S}}_{n}^{(j)}:=\left\{\pi \in \mathfrak{S}_{n}: \pi(j+1)=1\right\} \text { for } 1 \leqslant j<n \quad \text { and } \quad \overline{\mathfrak{S}}_{n}^{(n)}:=\left\{\pi^{\prime} \square 1: \pi^{\prime} \in \mathfrak{S}_{[n] \backslash\{1\}}\right\} .
$$

The " $\square$ " in $\pi=\pi_{1} \pi_{2} \cdots \pi_{n-1} \square 1 \in \overline{\mathfrak{S}}_{n}^{(n)}$ means that the $n$-th position of $\pi$ is empty and the hook factorization of $\pi$ is defined to be $p \tau_{1} \cdots \tau_{r} \square 1$, where $p \tau_{1} \cdots \tau_{r}$ is the hook factorization of $\pi_{1} \cdots \pi_{n-1}$ and " $\square 1$ " is viewed as a hook. We also define the statistics

$$
\operatorname{lec}\left(\pi_{1} \pi_{2} \cdots \pi_{n-1} \square 1\right)=\sum_{i=1}^{r} \operatorname{lec}\left(\tau_{i}\right) \quad \text { and } \quad \operatorname{inv}\left(\pi_{1} \pi_{2} \cdots \pi_{n-1} \square 1\right)=\operatorname{inv}\left(\pi_{1} \pi_{2} \cdots \pi_{n-1} 1\right) .
$$

For example, $\overline{\mathfrak{S}}_{3}^{(3)}=\{32 \square 1,23 \square 1\}$ with $\operatorname{lec}(32 \square 1)=1, \operatorname{lec}(23 \square 1)=0, \operatorname{inv}(32 \square 1)=3$, and $\operatorname{inv}(23 \square 1)=2$.

Lemma 12. For $1 \leqslant j \leqslant n, B_{n, k}^{(j)}(q)=\sum_{\substack{\pi \in \overline{\mathfrak{S}}_{n}^{(j)} \\ l e c(\pi)=k}} q^{(i n v-l e c) \pi}$.

Proof. Let $\bar{B}_{n}^{(j)}(t, q):=\sum_{\pi \in \overline{\mathfrak{S}}_{n}^{(j)}} q^{(\mathrm{inv}-\mathrm{lec}) \pi} t^{\text {lec } \pi}$. We recall that, to derive the hook factorization of a permutation, one can start from the right and factor out each hook step by step. Therefore, the hook factorization of $\pi=\pi_{1} \cdots \pi_{j-1} \pi_{j} 1 \pi_{j+2} \cdots \pi_{n}$ in $\pi \in \overline{\mathfrak{S}}_{n}^{(j)}$ is $p \tau_{1} \cdots \tau_{s} \tau_{1}^{\prime} \cdots \tau_{r}^{\prime}$, where $p \tau_{1} \cdots \tau_{s}$ and $\tau_{1}^{\prime} \cdots \tau_{r}^{\prime}$ are hook factorizations of $\pi_{1} \cdots \pi_{j-1}$ and $\pi_{j} 1 \pi_{j+2} \cdots \pi_{n}$, respectively. When $n>j$, it is not difficult to see that

$$
\operatorname{lec}\left(\pi_{j} 1 \pi_{j+2} \cdots \pi_{n}\right)=1+\operatorname{lec}\left(\pi_{j} \pi_{j+2} \cdots \pi_{n}\right)
$$

and

$$
(\mathrm{inv}-\mathrm{lec})\left(\pi_{j} 1 \pi_{j+2} \cdots \pi_{n}\right)=(\mathrm{inv}-\mathrm{lec})\left(\pi_{j} \pi_{j+2} \cdots \pi_{n}\right) .
$$

Thus by (2.3), we have

$$
\bar{B}_{n}^{(j)}(t, q)=A_{j-1}(t, q) q^{j-1}\left[\begin{array}{l}
n-1 \\
j-1
\end{array}\right]_{q} t A_{n-j}(t, q)
$$

for $n>j$. Clearly, $\bar{B}_{j}^{(j)}(t, q)=A_{j-1}(t, q) q^{j-1}$. So, by (1.2), the exponential generating function $\sum_{n \geqslant j} \bar{B}_{n}^{(j)}(t, q) \frac{z^{n-1}}{(q ; q)_{n-1}}$ is the right side of (1.8). This finishes the proof of the lemma.

Remark 6. This interpretation can also be deduced directly from the interpretation in Lemma 10 using Burstein's bijection [2].

For $X \subset[n]$ with $|X|=m$ and $1 \in X$, we can define $\overline{\mathfrak{S}}_{X}^{(j)}$ for $1 \leqslant j \leqslant m$ similarly as $\overline{\mathfrak{S}}_{m}^{(j)}$ like this:

$$
\overline{\mathfrak{S}}_{X}^{(j)}:=\left\{\pi \in \mathfrak{S}_{X}: \pi(j+1)=1\right\} \text { for } 1 \leqslant j<m \quad \text { and } \quad \overline{\mathfrak{S}}_{X}^{(m)}:=\left\{\pi^{\prime} \square 1: \pi^{\prime} \in \mathfrak{S}_{X \backslash\{1\}}\right\} .
$$

For $1 \leqslant j \leqslant n$, we define a $j$-restricted two-pix-permutation of $[n]$ to be a pair $\mathbf{v}=\left(\pi, p_{2}\right)$ satisfying: 
- $p_{2}$ (possibly empty) is an increasing word on $[n]$ and

- $\pi \in \overline{\mathfrak{S}}_{X}^{(j)}$ with $X=[n] \backslash \operatorname{cont}\left(p_{2}\right)$.

Similarly, we define $\operatorname{lec}(\mathbf{v})=\operatorname{lec}(\pi)$ and $\operatorname{inv}(\mathbf{v})=\operatorname{inv}(\pi)+\operatorname{inv}\left(\operatorname{cont}(\pi), \operatorname{cont}\left(p_{2}\right)\right)$. Let $\mathcal{W}_{n}^{(j)}$ denote the set of all $j$-restricted two-pix-permutations of $[n]$.

Lemma 13. Let $a, j$ be positive integers. Then

$$
\sum_{\substack{\mathbf{v} \in \mathcal{W}_{n}^{(j)} \\
\text { lecv }=a}} q^{(i n v-l e c) \mathbf{v}}=\sum_{k \geqslant 1}\left[\begin{array}{l}
n-1 \\
k-1
\end{array}\right]_{q} B_{k, a}^{(j)}(q) .
$$

Proof. It follows from Lemma 12 and some similar arguments as in the proof of Lemma 5.

Lemma 14. Let $2 \leqslant j \leqslant n$. Then there is an involution $\mathbf{v} \mapsto \mathbf{u}$ on $\mathcal{W}_{n}^{(j)}$ satisfying

$$
\operatorname{lec}(\mathbf{v})=n-2-\operatorname{lec}(\mathbf{u}), \quad \text { and }(i n v-l e c) \mathbf{v}=(i n v-l e c) \mathbf{u} .
$$

Proof. Suppose $\mathbf{v}=\left(\pi, p_{2}\right) \in \mathcal{W}_{n}^{(j)}$ and $\pi=\tau_{0} \tau_{1} \cdots \tau_{r}$ is the hook factorization of $\pi$ such that $\tau_{0}$ is a hook or an increasing word and $\tau_{i}(1 \leqslant i \leqslant r)$ are hooks. We also assume that $p_{2}=x_{1} \cdots x_{l}$ if $p_{2}$ is not empty. Note that $1 \notin \operatorname{cont}\left(\tau_{0}\right)$ since $j \neq 1$. We will use the involutions $d$ and $d^{\prime}$ defined in (2.4) and (2.5). There are several cases to be considered:

(i) $\tau_{r}=\square 1$. Then

$$
\mathbf{u}= \begin{cases}\left(d^{\prime}\left(\tau_{0}\right) d\left(\tau_{1}\right) \cdots d\left(\tau_{r-1}\right) x_{l} 1 x_{1} x_{2} \cdots x_{l-1}, \emptyset\right), & \text { if } p_{2} \neq \emptyset \\ \left(d^{\prime}\left(\tau_{0}\right) d\left(\tau_{1}\right) \cdots d\left(\tau_{r-1}\right) \square 1, \emptyset\right), & \text { otherwise }\end{cases}
$$

(ii) $\tau_{r}=y_{s} 1 y_{1} \cdots y_{s-1}$. Then

$$
\mathbf{u}= \begin{cases}\left(d^{\prime}\left(\tau_{0}\right) d\left(\tau_{1}\right) \cdots d\left(\tau_{r-1}\right) d\left(\tau_{r}\right) d^{\prime}\left(p_{2}\right), \emptyset\right), & \text { if } p_{2} \neq \emptyset ; \\ \left(d^{\prime}\left(\tau_{0}\right) d\left(\tau_{1}\right) \cdots d\left(\tau_{r-1}\right) \square 1, y_{1} \cdots y_{s}\right), & \text { if } p_{2}=\emptyset \text { and } y_{s}>y_{s-1} ; \\ \left(d^{\prime}\left(\tau_{0}\right) d\left(\tau_{1}\right) \cdots d\left(\tau_{r-1}\right) d^{\prime}\left(\tau_{r}\right), \emptyset\right), & \text { otherwise }\end{cases}
$$

(iii) $1 \notin \operatorname{cont}\left(\tau_{r}\right)$. Then

$$
\mathbf{u}= \begin{cases}\left(d^{\prime}\left(\tau_{0}\right) d\left(\tau_{1}\right) \cdots d\left(\tau_{r-1}\right) d\left(\tau_{r}\right) d^{\prime}\left(p_{2}\right), \emptyset\right), & \text { if } p_{2} \neq \emptyset ; \\ \left(d^{\prime}\left(\tau_{0}\right) d\left(\tau_{1}\right) \cdots d\left(\tau_{r-1}\right), d^{\prime}\left(\tau_{r}\right)\right), & \text { if } p_{2}=\emptyset \text { and } \operatorname{lec}\left(\tau_{r}\right)=\left|\tau_{r}\right|-1 ; \\ \left(d^{\prime}\left(\tau_{0}\right) d\left(\tau_{1}\right) \cdots d\left(\tau_{r-1}\right) d^{\prime}\left(\tau_{r}\right), \emptyset\right), & \text { otherwise. }\end{cases}
$$

First of all, one can check that $\mathbf{u} \in \mathcal{W}_{n}^{(j)}$. Secondly, as $d, d^{\prime}$ are involutions, the above mapping is an involution. Finally, this involution satisfies (4.7) in all cases. This completes the proof of the lemma.

Combining Lemmas 13 and 14 we obtain a bijective proof of Theorem 1. 


\section{Acknowledgement}

The author would like to thank Prof. Jiang Zeng for useful conversations.

\section{References}

[1] G.E. Andrews, On the foundations of combinatorial theory V, Eulerian differential operators, Stud. Appl. Math., 50 (1971), 345-375.

[2] A. Burstein, A combinatorial proof of joint equidistribution of some pairs of permutation statistics, talk at Permutation Patterns 2012.

[3] L. Carlitz, A combinatorial property of $q$-Eulerian numbers, Amer. Math. Monthly, 82 (1975), 51-54.

[4] C.-O. Chow, A recurrence relation for "inv" analogue of $q$-Eulerian polynomials, Electron. J. Combin., 17 (2010), \#N22.

[5] F. Chung and R. Graham, Generalized Eulerian Sums, J. Comb., 3 (2012), 299-316.

[6] F. Chung, R. Graham, D. Knuth, A symmetrical Enulerian identity, J. Comb., 1 (2010), 29-38.

[7] R.J. Clarke, E. Steingrímsson, and J. Zeng, New Euler-Mahonian statistics on permutations and words, Adv. in Appl. Math., 18 (1997), 237-270.

[8] D. Foata, On the Netto inversion number of a sequence, Proc. Amer. Math. Soc., 19 (1968), 236-240.

[9] D. Foata, Eulerian polynomials: from Euler's time to the present, The Legacy of Alladi Ramakrishnan in the Mathematical Sciences (2010), 253-273.

[10] D. Foata and G.-N. Han, The $q$-Tangent and $q$-Secant numbers via basic Eulerian polynomials, Proc. Amer. Math. Soc, 138 (2009), 385-393.

[11] D. Foata and G.-N. Han, Fix-mahonian calculus III; a quardruple distribution, Monatsh. Math., 154 (2008), 177-197.

[12] D. Foata and G.-N. Han, Decreases and descents in words, Sem. Lothar. Combin., B58a (2007), 17 pp.

[13] D. Foata and G.-N. Han, The decrease value theorem with an application to permutation statistics, Adv. in Appl. Math., 46 (2011), 296-311.

[14] I.M. Gessel, A coloring problem, Amer. Math. monthly, 98 (1991), 530-533.

[15] I.M. Gessel and C. Reutenauer, Counting permutations with given cycle structure and descent set, J. Combin. Theory Ser. A, 64 (1993), 189-215.

[16] G.-N. Han, Z. Lin and J. Zeng, A symmetrical $q$-Eulerian identity, Sem. Lothar. Combin., B67c (2012), 11 pp.

[17] G.-N. Han and G. Xin, Permutations with extremal number of fixed points, J. Combin. Theory Ser. A, 116 (2009), 449-459. 
[18] M. Hyatt, Eulerian quasisymmetric functions for the type B Coxeter group and other wreath product groups, Adv. in Appl. Math., 48 (2012), 465-505.

[19] D. Kim and J. Zeng, A new Decomposition of Derangements, J. Combin. Theory Ser. A, 96 (2001), 192-198.

[20] S. Linusson, J. Shareshian and M.L. Wachs, Rees products and lexicographic shellability, J. Comb., 3 (2012), 243-276.

[21] S. Park, The r-Multipermutations, J. Combin. Theory Ser. A, 67 (1994), 44-71.

[22] T.K. Petersen, Two-sided Eulerian numbers via balls in boxes, Math. Mag., to appear, 2012.

[23] J. Shareshian and M.L. Wachs, q-Eulerian polynomials: Excedance Number and Major Index, Electron. Res. Announc. Amer. Math. Soc. 13 (2007), 33-45.

[24] J. Shareshian and M.L. Wachs, Poset homology of Rees products, and $q$-Eulerian polynomials, Electron. J. Combin., 16 (2009), \#R20.

[25] J. Shareshian and M.L. Wachs, Eulerian quasisymmetric function, Adv. in Math., 225 (2011), 2921-2966.

[26] J. Shareshian and M.L. Wachs, Chromatic quasisymmetric functions and Hessenberg varieties, to appear in the Proceedings of De Giorgi Center Program on Configuration Spaces.

[27] R.P. Stanley, Enumerative combinatorics, Vol. 1, Cambridge University Press, Cambriage, 1997.

[28] R.P. Stanley, Binomial posets, Möbius inversion, and permutation enumeration, J. Combin. Theory Ser. A, 20 (1976), 336-356.

[29] M.L. Wachs, On q-derangement numbers, Proc. Amer. Math. Soc., 106 (1989), 273278. 